\title{
Angiosarcoma de mama. A propósito de un caso
}

\author{
F. J. Torres Gómez, J. Ibáñez Martínez, F. J. Torres Olivera
}

\section{Resumen}

El angiosarcoma es una neoplasia infrecuente en la mama si bien constituye uno de los sarcomas más frecuentes en esta localización existiendo en gran número de casos una estrecha relación entre su aparición y el antecedente de radioterapia a nivel torácico. Aunque los datos clínicos y las pruebas de imagen pueden orientar el diagnóstico, será necesario el estudio histológico e inmunohistoquímico para determinar el mismo. El pronóstico está determinado por el grado histológico, constituyendo las lesiones de alto grado un grupo con muy mal pronóstico. Presentamos un caso de angiosarcoma de mama no relacionado con radioterapia previa y de crecimiento insidioso que histológicamente mostraba patrones de bajo, moderado y alto grado.

Palabras clave: Angiosarcoma. Mama. Radioterapia.

Oncología, 2006; 29 (2):85-89

\section{Summary}

Angiosarcoma of the breast is a rare neoplasm but one of the most common sarcomas in this location, frequently associated with thoracic radiotherapy. Clinical data and imaging techniques may help the diagnosis, but histological and immunohistochemical studies become necessary in most cases. The prognosis depends on the histological grade, neoplasms of high grade having a worse prognosis. We report the case of a slow growth and variegate histological grade breast angiosarcoma having no relationship with radiotherapy.

Key words: Angiosarcoma. Breast. Radiotherapy.

Departamento de Anatomía Patológica Hospital Universitario Virgen Macarena Sevilla (España) 


\section{Introducción}

El angiosarcoma es una neoplasia maligna relativamente infrecuente a nivel mamario constituyendo menos del $0,05 \%$ de los tumores primarios de la mama si bien es uno de los sarcomas más frecuentes en esta localización ${ }^{1-3}$. Se trata de una neoplasia muy agresiva cuyo comportamiento biológico sigue un curso paralelo al grado de diferenciación de forma que cuanto menor sea el grado de diferenciación mayor será la agresividad tumoral. Existe una fuerte relación con el tratamiento radioterápico de la pared torácica, situación frecuente hoy día con la tendencia conservadora que el tratamiento del cáncer de mama está adquiriendo por lo cual no es de extrañar que en los años venideros asistamos a un aumento del número de estas neoplasias.

Si bien los criterios clínicos y los estudios de imagen pueden conducir a un diagnóstico de sospecha, únicamente el estudio histológico detallado (junto con el apoyo de las técnicas inmunohistoquímicas en casos en que sean necesarias) determinará el correcto diagnóstico de la lesión y su correcta gradación con la significación pronóstica que esta última conlleva.

Consideramos interesante la presentación de nuestro caso debido a la ausencia de antecedentes de radioterapia previa a la aparición de la lesión así como al debut clínico atípico del mismo.

\section{Caso clínico}

Paciente de 50 años sin antecedentes personales de interés que notaba desde hace tiempo un nódulo indurado a nivel de cuadrante inferior interno de mama izquierda. En la mamografía se observó una lesión irregular de límites imprecisos la cual condicionó la realización de una PAAF, que mostró una lesión fusocelular con atipia sobre fondo hemático. Con todo ello se decidió intervenir a la paciente.

Se recibió para estudio anatomopatológico un fragmento irregular de tejido fibroadiposo que mide $5 \times 3,9 \times 2 \mathrm{~cm}$ de dimensión máxima. A los cortes seriados se observó un área irregular ligeramente estrellada y blanquecina de $2 \mathrm{~cm}$ de diámetro máximo centrada por una cavidad quística de contornos anfractuosos y contenido pardusco y friable de 0,9 $\mathrm{cm}$ en torno a la cual se apreciaba un delicado punteado rojizo.

Histológicamente se realizó el diagnóstico de angiosarcoma mamario pudiéndose distinguir tres patrones relativamente bien diferenciados:

a) bajo grado: canales vasculares anastomosados cuyo endotelio muestra una única hilera celular con escasa o nula atipia, que infiltran el estroma y el tejido adiposo mamarios.

b) grado intermedio: aparecen pequeñas papilas en las luces vasculares junto con áreas de proliferación endotelial. Existen focos con atipia celular, mitosis y elementos celulares fusiformes, si bien éstos no son llamativos.

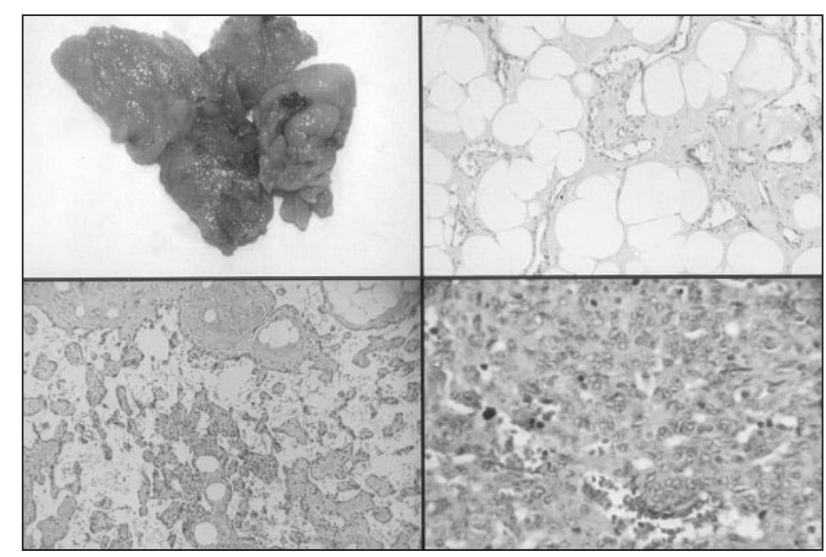

Figura 1. Angiosarcoma de mama. A) Imagen superior izquierda: aspecto macroscópico. B) Imagen superior derecha: áreas de bajo grado. C) Imagen inferior izquierda: Áreas de grado intermedio. D) Imagen inferior derecha: áreas de alto grado.

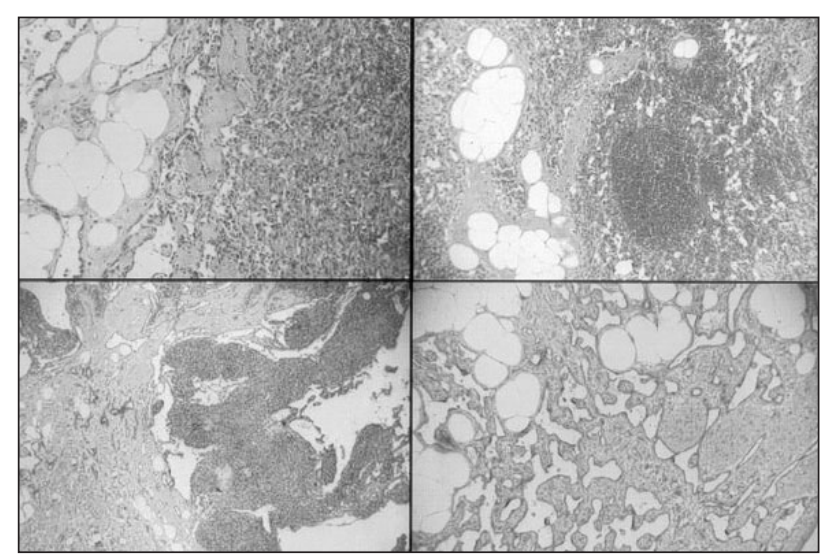

Figura 2. Angiosarcoma de mama. A) Imagen superior izquierda: áreas de alto y bajo Grado. B) Imagen superior derecha: lagos hemáticos. C) Imagen inferior izquierda: áreas de alto grado con patrón sólido. D) Imagen inferior derecha: Inmunotinción vascular con CD31. 
c) alto grado: llamativas proliferaciones papilares intravasculares de células endoteliales, áreas fusocelulares y extensas áreas de crecimiento sólido en que se distinguen focos necróticos y evidentes lagos vasculares. La atipia y las figuras de mitosis son prominentes.

La presencia de áreas de alto grado con las características histológicas expuestas condicionaron la asignación global de alto grado a la lesión pues son las mismas las que determinan la agresividad y por tanto el pronóstico de la neoplasia.

La lesión contactaba con los márgenes quirúrgicos de resección por lo que se llevó a cabo una mastectomía (tratamiento de elección del angiosarcoma mamario), observándose una lesión blanquecina estrellada de $4 \mathrm{~cm}$ de dimensión máxima así como varios nódulos de similares características ambos en contacto con la cavidad de tumorectomía previa y correspondiendo con áreas de angiosarcoma residual y áreas de necrosis grasa respectivamente. No se objetivaron adenopatías. La paciente recibió varios ciclos de radioterapia postoperatoria y fue seguida por el Servicio de Oncología durante el periodo de dieciocho meses, ingresando al cabo de este periodo en el Servicio de Urgencias debido a coma hepático, muriendo cuatro días después de tal ingreso. Estudios de imagen subsiguientes (TAC) demostraron múltiples metástasis hepáticas.

\section{Discusión}

Los sarcomas son infrecuentes en la mama ( $1 \%$ de tumores malignos), si bien el angiosarcoma muestra cierta preferencia por ella en comparación con otros órganos ${ }^{3,4}$. Ello queda reflejado en los múltiples casos aislados descritos en la literatura y las escasas series de los mismos. Son neoplasias que aparecen a cualquier edad con mayor incidencia en mujeres adultas jóvenes, generalmente como nódulos asintomáticos detectados por la paciente en exploraciones rutinarias si bien en ocasiones su crecimiento es excesivo llegando a observarse una gran masa mamaria con coloración azulada de la piel suprayacente ${ }^{5}$. Varios estudios han intentado demostrar una etiología hormonal basándose en su mayor frecuencia en el embarazo. Otros trabajos relacionan su aparición con los tratamientos radioterápicos a nivel torácico. Esta relación ha sido demostrada en pacientes con antecedentes de carcinoma de mama que han sido sometidas a tratamiento conservador asociado a radioterapia ${ }^{6,7}$ (en tal caso el angiosarcoma ha sido descrito tanto a nivel del parénquima mamario como a nivel cutáneo) o bien a mastectomía (en este caso existe un alto número de lesiones angiosarcomatosas descritas a nivel de la pared costal). En ambos casos existe un periodo de latencia variable con una media de unos 6-7 años para su aparición. La relación con la radioterapia es tan importante que podemos clasificar a los angiosarcomas mamarios en tres grupos clínicos bien diferenciados:

- Angiosarcomas primarios, que pueden aparecer a cualquier edad si bien las mujeres de 30-40 años constituyen el grupo más numeroso.

- Angiosarcomas postradioterapia, que afectan a un grupo de edad superior, principalmente postmenopausicas ${ }^{8}$.

- Angiosarcomas asociados a Síndrome de Stewart-Treves, con linfedema de larga evolución, hoy día menos frecuente debido a la tendencia conservadora de la cirugía.

Si bien los datos clínicos pueden orientar hacia la etiología en algunas ocasiones, será el estudio histológico el que determine el diagnóstico definitivo. La radiología aporta datos como el tamaño, delimitación y heterogeneidad pero no sirve para concluir con el diagnóstico de angiosarcoma9 ${ }^{9}$ También es relativa la ayuda de la PAAF en la cual se pueden observar grupos de células fusiformes, inespecíficos en la mayoría de ocasiones para el diagnóstico de angiosarcoma si bien estos hallazgos podrían dirigir nuestras sospechas hacia el diagnóstico de sarco$\mathrm{ma}^{10}$. Desde el punto de vista macroscópico suelen ser lesiones irregulares que miden más de $5 \mathrm{~cm}$ de dimensión máxima y muestran al corte una tonalidad pardo-rojiza y en ocasiones aspecto necrótico.

Histológicamente se suele tratar de lesiones irregulares con márgenes infiltrativos pudiéndose observar distintos patrones histológicos, como en nuestro caso, aunque generalmente predomina uno de ellos sobre los demás. Es importante hacer un estudio seriado del tumor pues se trata de neoplasias tremendamente agresivas con alto poder metastatizante (pulmón, hueso, mama contralateral, hígado, piel, etc) y el grado histológico es el factor pronóstico más importante. 
En el caso que presentamos se han podido observar los tres patrones histológicos descritos si bien su distribución zonal es relativamente uniforme de forma que las áreas de bajo grado se concentran principalmente en la periferia mientras que aquellas sólidas de alto grado muestran una distribución principalmente central. Ello podría justificar el peculiar patrón de crecimiento de la lesión y la ausencia de nódulo cutáneo azulado, factores que hubiesen contribuido a sospechar el diagnóstico clínicamente.

El diagnóstico diferencial hay que establecerlo principalmente con otras lesiones vasculares mamarias, principalmente con hemangiomas y angiomatosis, entidades con características similares a las de otras localizaciones en las que sólo un estudio exhaustivo logra con dificultad diferenciarlas de las lesiones de bajo grado, sobre todo en el caso de los denominados hemangiomas atípicos, los cuales muestran hipercromatismo nuclear en una lesión arquitecturalmente benigna. Las lesiones de grado intermedio deben diferenciarse de las hiperplasias papilares endoteliales de localización intravascular las cuales, a diferencia de los angiosarcomas, se encuentran bien delimitadas y/o encapsuladas y se asocian a material trombótico. Aún más complicado es el diagnóstico diferencial de los angiosarcomas con predominio del componente fusocelular con lesiones mesenquimales fusocelulares de agresividad local (fibromatosis), con carcinomas sarcomatoides y con auténticos sarcomas mamarios donde será necesario en la mayoría de las ocasiones realizar estudios inmunohistoquímicos para demostrar el componente vascular de la lesión. Finalmente se debe tener también en cuenta la hiperplasia pseudoangiomatosa del estroma mamario, entidad en la que existe una proliferación miofibroblástica delimitando espacios en los que se pueden depositar mucipolisacáridos.

Los angiosarcomas mamarios han demostrado ser un grupo heterogéneo desde el punto de vista genético ${ }^{11}$ habiéndose observado diversas alteraciones cromosómicas entre las que destacan por su frecuencia las ganancias de $8 \mathrm{q}$ y $20 \mathrm{p}$ y las pérdidas de $5 \mathrm{q}$ y $16 \mathrm{q}^{11}$.

Los angiosarcomas se incluyen dentro del grupo de las más agresivas neoplasias de localización mamaria debido a la temprana aparición de metástasis y a la alta tasa de recidivas siendo la mortalidad de hasta el 50\% a los dos años del diagnóstico. El gra- do histológico, como hemos comentado anteriormente, matizará un pronóstico de por sí ominoso. La mastectomía es el procedimiento terapéutico de elección habiéndose descrito recurrencias en la mayoría de pacientes a los que se les ha aplicado cirugía conservadora. Esta última se sigue practicando como primer escalón en la escala terapéutica en casos que, como el nuestro, presentan una clínica en esencia similar a la de otras neoplasias mamarias y únicamente aquellas pacientes a las que se les ha realizado una biopsia o PAAF diagnósticas acudirán al quirófano con un diagnóstico definitivo que guíe al cirujano en su elección terapéutica. Se beneficiarán de la mastectomía principalmente aquellas pacientes que sean intervenidas temprano sin crecimiento lesional excesivo. Generalmente no se realizará linfadenectomía axilar debido a la escasa tendencia de la neoplasia a utilizar la vía linfática para metastatizar; aquella quedará restringida a los casos en que se constate linfadenopatía mediante exploración clínica. También se ha demostrado la efectividad de la quimioterapia (dactinomicina) en el tratamiento de algunas pacientes si bien el beneficio de los distintos tratamientos quimioterápicos no está bien establecido.

\section{Conclusiones}

Debería sospecharse angiosarcoma en aquellas lesiones de mama que debutan clínicamente con un nódulo cutáneo azulado de crecimiento relativamente rápido sobre todo en aquellos casos en que existe antecedente de radioterapia previa si bien existen lesiones como la aquí expuesta que presentan una clínica que en esencia no difiere de la de otros tumores mamarios. En cualquier caso es conveniente realizar PAAF ante todo nódulo mamario pues la simple observación de una rica población fusocelular con o sin atipia puede orientar un diagnóstico temprano. El estudio histológico (biopsia preoperatoria, intraoperatoria, tumorectomía o mastectomía) determinarán el grado y por tanto el pronóstico de la lesión. La mastectomía es el tratamiento de elección acompañada o no de quimioterapia coadyuvante, cuyos beneficios están aún por definir.

Recomendamos estar alerta y tener un alto grado de sospecha ante una neoplasia cuya incidencia se está incrementando día a día paralelamente a la 
tendencia conservadora del tratamiento del cáncer mamario en la actualidad. Asimismo recordamos que se trata de una enfermedad tremendamente agresiva que requiere necesariamente un diagnóstico precoz.
Correspondencia:

Dr. F. J. Torres Gómez

Plaza de la Encarnación, 32 - P1- $2^{\circ} \mathrm{B}$

E-41003 Sevilla

javiertorresgomez@yahoo.es

\section{Bibliografía}

1. Hunter TB, Martin PC, Dietzen CD, Tyler LT. Angiosarcoma of the breast. Two case reports and a review of the literature. Cancer 1985; 56:2099-106.

2. Mark RJ, Poen JC, Tran LM, Fu YS, Juillard GF. Angiosarcoma. A report of 67 patients and a review of the literature. Cancer 1996; 77:2400-6.

3. Paul Peter Rosen, MD, Harold A. Oberman, MD. Tumors of the Mammary Gland. Atlas of Tumor Pathology. AFIP 1993.

4. Kondis-Pafitis A, Psyhogios J, Spanidou-Carvouni H. Clinicopathological study of vascular tumors of the breast: a series of ten patients with long follow up. Eur J Gynaecol Oncol 2004; 25(3):324-26.

5. WynnGR, Bentley PG, Liebman R, Fletcher CD. Mammary parenchymal angiosarcoma after breast conserving treatment for invasive high grade ductal carcinoma. Breast J 2004 Nov-Dec; 10(6):558-59.

6. Billings SD, McKenney JK, Folpe AL, Hardacre MC, Weiss SW. Cutaneous angiosarcoma following breast-conserving surgery and radiation: an analysis of 2-7 cases. Am J Surg Pathol 2004 Jun; 28(6):781-8.

7. Parker RG, Barsky SH, Bennion R. Angiosarcoma developing in a breast after conservation treatment for breast cancer. Am J Clin Oncol 2003 Oct; 26(5):486-8.

8. Parker RG, Barsky SH, Bennion R. Angiosarcoma developing in a breast after conservation treatment for breast cancer. Am J Clin Oncol 2003 Oct; 26(5):486-8.

9. Johnson GM, Garguilo GA. Angiosarcoma of the breast: a case report and literature review. Curr Surg 2002 Sep-Oct; 59(5):490-94.

10. Gherardi G, Rossi S, Perone S, Scanni A. Angiosarcoma after breast-conserving therapy: fine needle aspiration biopsy, immunocytochemistry and clinicopathologic correlates. Cancer 2005 Jun 25; 105(3):145-51.

11. Baumhoer D, Gunawan B, Becker H, Fuzesi L. Comparative genomic hybridization in four angiosarcomas of the female breast. Gynecol Oncol 2005 May; 97(2):348-52. 